\title{
EXPLICIT KARHUNEN-LOĖVE EXPANSIONS RELATED TO THE GREEN FUNCTION OF THE LAPLACIAN
}

\author{
J.-R. PYCKE \\ L.S.T.A, Jean-Renaud Pycke, Université Paris VI \\ case 158, 175 rue du Chevaleret, 75013 Paris, France \\ E-mail:pycke@ccr.jussieu.fr
}

\begin{abstract}
Karhunen-Loève expansions of Gaussian processes have numerous applications in Probability and Statistics. Unfortunately the set of Gaussian processes with explicitly known spectrum and eigenfunctions is narrow. An interpretation of three historical examples enables us to understand the key role of the Laplacian. This allows us to extend the set of Gaussian processes for which a very explicit Karhunen-Loève expansion can be derived.
\end{abstract}

1. Introduction. Karhunen-Loève (or K-L) expansions of Gaussian processes are of great interest in Statistics and Probability. We shall recall briefly their definition and give a few examples of their applications in fields as large deviations and small balls probabilities, Cramér-von Mises type and U- or V-statistics. We will be concerned with their statistical applications. One of the main problems in this field is the difficulty to obtain explicit K-L expansions. We give in Section 2 a geometrical interpretation of the two well-known expansions (1) and (2), in terms of the Green's function of the Laplacian on the circle. These two kernels are related to the celebrated Watson's and Cramér-von Mises statistics. Thus the interpretation we propose opens the way to generalizations of these statistics to other manifolds, outlined in Section 3. The key idea is to consider Green's function of the Laplacian on a given manifold as the kernel of a U-statistic suited for a test of uniformity on this manifold. Proposition 3.1 gives an explicit expression for Green's function of the Laplacian of compact, 2-point homogeneous spaces as Euclidean spheres, real complex and quaternion projective spaces. In these spaces an averaging process applied to the Green's function K-L expansion (6) enables one to obtain a second kernel and its K-L expansion, which can be viewed as its zonal or radial part. Thus the K-L expansions (1) - (2) arise as a particular case of the expansions $(6)-(7)$. The case of the 2-dimensional unit sphere is discussed in Section 4. We show that the kernel of the

2000 Mathematics Subject Classification: 62G10, 62E20.

The paper is in final form and no version of it will be published elsewhere. 
Anderson-Darling statistic viewed as a V-statistic is the radial part of Green's function. The U-statistic whose kernel is Green's function of the sphere is introduced in Theorem 1. It is the analogue on the sphere of Watson's statistic which has been introduced in [Wat61] for the circle. Interestingly, this new statistic has a simple expression in terms of the geometrical mean of the sample spacings.

We use the following notations. Let $T \subseteq \mathbb{R}^{n}$ and $\{\mathbf{X}(t): t \in T\}$ denote a centered Gaussian process on the measure space $(T, \mu)$ with covariance function

$$
K(s, t):=\mathbf{E X}(s) \mathbf{X}(t) \quad \text { such that } \int_{T \times T} K^{2}(s, t) d \mu(s) d \mu(t)<\infty .
$$

The Karhunen-Loève expansion is

$$
K(s, t)=\sum_{k=1}^{\infty} \frac{f_{k}(s) f_{k}(t)}{\lambda_{k}} \quad \text { or } \quad \mathbf{X}(t) \stackrel{(\text { law })}{=} \sum_{k=1}^{\infty} \frac{\xi_{k} f_{k}(t)}{\sqrt{\lambda_{k}}}
$$

where the $\xi_{k}$ are independent $\mathcal{N}(0,1)$ random variables and the sequence $\left\{\left(\lambda_{k}, f_{k}\right): k \geq\right.$ $1\}$ is a complete set of solutions of the integral equation

$$
\lambda_{k} \int_{T} K(s, t) f_{k}(s) d \mu(s)=f_{k}(t) \quad \text { with } \quad \int_{T} f_{k}(s) f_{\ell}(s) d \mu(s)= \begin{cases}0 & \text { if } k \neq \ell \\ 1 & \text { if } k=\ell .\end{cases}
$$

Interesting by-products of the explicit K-L expansion concern the study of the $L^{2}$ norm of X. Firstly (see [SW86] formula (14) p. 212),

$$
\begin{gathered}
\int_{T} \mathbf{X}^{2}(t) d \mu(t) \stackrel{(\text { law })}{=} \sum_{k=1}^{\infty} \frac{\xi_{k}^{2}}{\lambda_{k}} \text { so that } \\
\mathbf{E} \exp \left\{i u \int_{T} \mathbf{X}^{2}(t) d \mu(t)\right\}=\prod_{k=1}^{\infty}\left(1-\frac{2 i u}{\lambda_{k}}\right)^{-\frac{1}{2}},(u \in \mathbb{R}) .
\end{gathered}
$$

Secondly for large deviations one has

$$
P\left(\int_{0}^{1} \mathbf{X}^{2}(t) d \mu(t) \geq x^{2}\right) \sim \frac{\left(\frac{\lambda_{1} x^{2}}{2}\right)^{\frac{n_{1}}{2}-1} e^{-\frac{\lambda_{1} x^{2}}{2}}}{\Gamma\left(\frac{n_{1}}{2}\right) \prod_{k=n_{1}+1}^{\infty}\left(1-\frac{\lambda_{1}}{\lambda_{k}}\right)} \quad \text { as } x \rightarrow \infty
$$

when the smallest eigenvalue $\lambda_{1}$ has multiplicity $n_{1}$ (see [Zol61] p. 204). Finally for the problem of small balls which is most of the times more involved, see e.g. [LS01] and the numerous references therein. One of the properties we infer from the K-L expansion is, as $\varepsilon \rightarrow 0$,

$$
\sum_{k=1}^{\infty}\left|\frac{c(k+d)^{2}}{\lambda_{k}}-1\right|<\infty \text { with } c>0, d \in \mathbb{R} \Rightarrow \log P\left(\int_{0}^{1} \mathbf{X}^{2}(t) d \mu(t) \leq \varepsilon^{2}\right) \sim-\frac{\pi^{2}}{8 c \varepsilon^{2}} .
$$

Statisticians use K-L expansions in the study of two families of statistics: Cramér-von Mises type statistics and U- or V-statistics. For details, see respectively [SW86], Chapter 5 and $[\mathrm{KB} 94] \S 4.3$. The statistician rejects the hypothesis

$\mathbf{H}_{\mathbf{0}}: X_{1}, \ldots, X_{n}$ are i.i.d. random variables with distribution $\mu$ on $T$ for large values of the $\mathrm{V}$-statistic $S_{n}:=\frac{1}{n} \sum_{i, j}^{n} K\left(X_{i}, X_{j}\right)$, of the U-statistic $S_{n}^{\prime}:=$ 
$\frac{2}{n-1} \sum_{i<j}^{n} K\left(X_{i}, X_{j}\right)$ or of the Cramér-von Mises type statistic

$$
T_{n}:=\int_{T} E_{n}^{2}\left[X_{1}, \ldots, X_{n}\right](t) d \mu(t)
$$

where $K: T \times T \rightarrow \mathbb{R}$ is a kernel and $E_{n}$ an empirical process converging in $L^{2}$ toward a centered Gaussian process $\mathbf{X}$. Given the K-L expansion of $K$ or $\mathbf{X}$, under $H_{0}$,

$$
\begin{gathered}
S_{n} \rightarrow \sum_{k=1}^{\infty} \frac{\xi_{k}^{2}}{\lambda_{k}}, S_{n}^{\prime} \rightarrow \sum_{k=1}^{\infty} \frac{\xi_{k}^{2}-1}{\lambda_{k}} \text { under the degeneracy condition } \int_{T} K(x, y) d \mu(y)=0, \\
\text { or } T_{n} \rightarrow \int_{T} \mathbf{X}^{2}(t) d \mu(t)=\sum_{k=1}^{\infty} \frac{\xi_{k}^{2}}{\lambda_{k}} .
\end{gathered}
$$

This means that the asymptotic distribution of $S_{n}, S_{n}^{\prime}$ and $T_{n}$ can be obtained from a K-L expansion. Unfortunately, as mentioned above, very few explicit K-L expansions useful in Statistics can be derived. We now describe an approach allowing one to obtain new explicit expansions.

2. The case of the circle revisited. We will revisit the historical examples of the Watson $\left(U_{n}^{2}\right)$ and Cramér-von Mises $\left(W_{n}^{2}\right)$ statistics. For further use in Section 4, we also recall results concerning the Anderson-Darling statistic $\left(A_{n}^{2}\right)$. These three statistics are widely used to test whether the sample $x_{1}, \ldots, x_{n}$ comes from the uniform distribution on $(0,1)$. For their definition, see [Dur73], formulas $(4.1 .7)-(4.1 .8)$ p. 27 and (5.4.2) p. 36 or [DS86], formulas (4.2) p. 101. Elementary computations enable to express them in the form of the V-statistics (for which we give the K-L expansion of the kernel)

$$
U_{n}^{2}=\frac{1}{n} \sum_{i=1}^{n} \sum_{j=1}^{n}\left\{\frac{\left(\left|x_{i}-x_{j}\right|-1 / 2\right)^{2}}{2}-\frac{1}{24}\right\} \quad \text { with the kernel }
$$

$K_{U}\left(x_{1}, x_{2}\right):=\frac{\left(\left|x_{1}-x_{2}\right|-1 / 2\right)^{2}}{2}-\frac{1}{24}$

$$
=\sum_{k=1}^{\infty} \frac{2 \sin \left(2 k \pi x_{1}\right) \sin \left(2 k \pi x_{2}\right)+2 \cos \left(2 k \pi x_{1}\right) \cos \left(2 k \pi x_{2}\right)}{4 k^{2} \pi^{2}}
$$

$$
W_{n}^{2}=\frac{1}{n} \sum_{i=1}^{n} \sum_{j=1}^{n}\left\{\frac{x_{i}^{2}-x_{i}+x_{j}^{2}-x_{j}-\left|x_{i}-x_{j}\right|}{2}+\frac{1}{3}\right\} \quad \text { with }
$$

$$
K_{W}\left(x_{1}, x_{2}\right):=\frac{x_{1}^{2}-x_{1}+x_{2}^{2}-x_{2}-\left|x_{1}-x_{2}\right|}{2}+\frac{1}{3}=\sum_{k=1}^{\infty} \frac{2 \cos \left(k \pi x_{1}\right) \cos \left(k \pi x_{2}\right)}{k^{2} \pi^{2}} \text {; }
$$

$$
A_{n}^{2}=\frac{1}{n} \sum_{i=1}^{n} \sum_{j=1}^{n}\left\{-\log \left[\max \left(x_{i}, x_{j}\right)-x_{i} x_{j}\right]-1\right\} \quad \text { with }
$$

$$
K_{A}\left(x_{1}, x_{2}\right):=-\log \left[\max \left(x_{1}, x_{2}\right)-x_{1} x_{2}\right]-1=\sum_{k=1}^{\infty} \frac{(2 k+1) p_{k}\left(x_{1}\right) p_{k}\left(x_{2}\right)}{k(k+1)}
$$

where $p_{k}$ is a Legendre polynomial, defined by (8). 
The Watson and Cramér-von Mises statistics can be interpreted in terms of the zeromean Green function $G_{1}$ of the Laplacian on the circle $S^{1}(R):=\left\{P(x)=R e^{2 i \pi x}: x \in\right.$ $\mathbb{R}\}$ as follows. Watson's statistic was introduced in order to test whether the sample $P_{1}:=P\left(x_{1}\right), \ldots, P_{n}:=P\left(x_{n}\right)$ comes from the uniform distribution on $S^{1}(R)$, on which the density of the Riemannian measure will be denoted by $d P$. We fix a north pole $N:=P(0)$, to which corresponds the south pole $P(1 / 2)$. Let $s: P \mapsto s \cdot P$ denote the reflection in the axis passing through the poles. For $x, y \in[0,1]$, the distance between the two points $P(x)$ and $Q(y)$ is $d(P, Q)=\min (2 \pi R|x-y|, 2 \pi R(1-|x-y|))$. In this setting, elementary computations lead to the interesting expressions

$$
\begin{aligned}
2 \pi R K_{U}\left(x_{1}, x_{2}\right) & =G_{1}\left(P_{1}, P_{2}\right), \\
\frac{\pi R}{2} K_{W}\left(\frac{d\left(P_{1}, N\right)}{\pi R}, \frac{d\left(P_{2}, N\right)}{\pi R}\right) & =\frac{G_{1}\left(P_{1}, P_{2}\right)+G_{1}\left(P_{1}, s \cdot P_{2}\right)}{2}
\end{aligned}
$$

where the function

$$
G_{1}\left(P_{1}, P_{2}\right):=\frac{\left[\pi R-d\left(P_{1}, P_{2}\right)\right]^{2}}{4 \pi R}-\frac{\pi R}{12}
$$

satisfies

$$
\left\{\begin{array}{l}
\left(-\Delta_{Q}\right) G_{1}(P, Q)=\delta_{P}-\frac{1}{2 \pi R}, \\
\int_{S^{1}(R)} G_{1}(P, Q) d Q=0 .
\end{array}\right.
$$

where $\delta_{P}$ denotes the Dirac distribution at the point $P$. In other words, Watson's kernel is the zero-mean Green's function of the Laplacian on $S^{1}(R)$, while Cramér-von Mises kernel is its radial part obtained by an averaging process. By the radial part of a function defined on a space with a fixed pole $N$, we mean the function taking at $P$ the average value of the function on the sphere centered at $N$ and containing $P$. In the present case, on the circle, this sphere consists of two points $P$ and $s \cdot P$.

3. Generalization to 2-point homogeneous spaces. From [Aub82], Definition (14) p. 107 (up to the opposite sign convention used for the Laplacian in this reference) and assertion (e) in Theorem 4.13 p. 108, we know that on any finite dimensional, $C^{\infty}$, compact manifold $M$ without boundary, there exists a zero-mean Green's function of the Laplacian on $M$. It satisfies (4) in which $S^{1}(R)$ is replaced by $M$ and $2 \pi R$ changed into $V_{M}$, the volume of $M$. Green's function is defined up to a constant. We choose the Green's function so that its integral equals zero, because this condition corresponds to the degeneracy condition required from the kernel of a degenerate U-statistic. In the same way, for statistical purposes, our aim is to obtain explicit expressions, not only existence results for the Green's function. For this reason, we are interested by manifolds for which an explicit expression of Green's function is available. Among them, the family of compact 2-point homogeneous spaces fulfills this restrictive condition. A 2-point homogeneous space $M$ is such that given any two pairs of points $P_{1}, Q_{1}, P_{2}, Q_{2}$ satisfying $d\left(P_{1}, Q_{1}\right)=$ $d\left(P_{2}, Q_{2}\right)$, there exists an isometry $\iota: M \rightarrow M$ such that $\iota\left(P_{1}\right)=P_{2}$ and $\iota\left(Q_{1}\right)=Q_{2}$. By virtue of Wang's classification in [Wan52], 2-point homogeneous spaces, compact and without boundary, are the Euclidean spheres, the real, complex and quaternion projective spaces, the Cayley plane (this exhaustive list is also given in [Hel00] p. 167). In the sequel, 
$M$ will denote one of these spaces. The Green's function on $M$ can be computed in terms of the volume $V(r)$ and the area $A(r)$ of a geodesic ball of radius $r$.

Proposition 3.1. The zero-mean Green's function of the Laplacian on $M$ is given by

$$
G_{M}\left(P_{1}, P_{2}\right)=\frac{1}{V_{M}} \int_{d\left(P_{1}, P_{2}\right)}^{\delta} \frac{V_{M}-V(r)}{A(r)} d r-\frac{1}{V_{M}^{2}} \int_{0}^{\delta} \frac{V(r)\left[V_{M}-V(r)\right]}{A(r)} d r
$$

where $\delta$ is the diameter of the space.

Proof. We use Theorem 5.27.(ii) p. 316, Chapter II in [Hel00]. The function

$$
-G(P, Q):=\left(1 / V_{M}\right) \int_{d(P, Q)}^{\delta} \frac{V_{M}-V(r)}{A(r)} d r
$$

introduced in this theorem is a Green's function of the Laplacian. A Green's function is defined up to a constant and the one appearing in our expression of $G_{M}$ is such that $\int_{M} G_{M}(P, Q) d Q=0$. It is obtained from the equalities

$$
\begin{aligned}
\int_{M} G(P, Q) d Q & =\int_{0}^{\delta}\left\{\int_{r}^{\delta}\left(\frac{V(\rho)-V_{M}}{V_{M} A(\rho)}\right) d \rho\right\} A(r) d r \\
& =\iint_{[0, \delta] \times[0, \delta]} \frac{V(\rho)-V_{M}}{V_{M} A(\rho)} A(r) 1_{\{r \leq \rho\}} d \rho d r \\
& =\int_{0}^{\delta} \frac{V(\rho)-V_{M}}{V_{M} A(\rho)}\left(\int_{0}^{\rho} A(r) d r\right) d \rho=\int_{0}^{\delta} \frac{V(\rho)\left[V(\rho)-V_{M}\right]}{V_{M} A(\rho)} d \rho
\end{aligned}
$$

We fix a pole $N \in M$, and denote by $\left(\lambda_{\ell}\right)_{\ell \geq 1}$ the increasing sequence of pairwise distinct positive eigenvalues of the opposite of the Laplacian $\left(-\Delta_{M}\right)$. Each eigenspace $E_{\ell}$ associated to $\lambda_{\ell}$ is of finite dimension, and has an orthonormal basis $\left\{f_{\ell}^{k}: 0 \leq k<\right.$ $\left.\operatorname{dim} E_{\ell}\right\}$ defined as follows. $f_{\ell}^{0}$ is a zonal spherical function, i.e. $f_{\ell}^{0}(P)$ depends only on $r=d(P, N)$, hence we will write $f_{\ell}^{0}(P)=f_{\ell}^{0}(r)$. For each $k \geq 1, f_{\ell}^{k}$ is an associated spherical function, i.e. of mean zero on each sphere of center $N$. The K-L expansion of $G_{M}$ (in a distributional sense) takes the form

$$
G_{M}\left(P_{1}, P_{2}\right)=\underbrace{\sum_{\ell \geq 1} \frac{f_{\ell}^{0}\left(r_{1}\right) f_{\ell}^{0}\left(r_{2}\right)}{\lambda_{\ell}}}_{\text {zonal expansion }}+\underbrace{\sum_{k, \ell \geq 1} \frac{f_{\ell}^{k}\left(P_{1}\right) f_{\ell}^{k}\left(P_{2}\right)}{\lambda_{\ell}}}_{\text {associated expansion }}
$$

Given $P_{1}$, the average of $G_{M}\left(P_{1}, Q\right)$ on a sphere of center $N$ and radius $r_{2}$ has consequently the bilinear expansion

$$
G_{M}^{\mathcal{M}}\left(r_{1}, r_{2}\right)=\sum_{\ell \geq 1} \frac{f_{\ell}^{0}\left(r_{1}\right) f_{\ell}^{0}\left(r_{2}\right)}{\lambda_{\ell}} .
$$

Furthermore explicit expressions for $V(r), A(r)$, the zonal spherical functions and the spectrum of the Laplacian can be found for 2-point homogeneous spaces (see [Gra73] examples 1-4 p. 340-341, [Ask75] p. 65, [Bes78] p. 201). This enables us to envisage the possibility of constructing a $\mathrm{U}$ - and a $\mathrm{V}$-statistic with kernel $G_{M}$ and $G_{M}^{\mathcal{M}}$ respectively. 
Their asymptotic distribution under the null-hypothesis will follow from (6) and (7). In order to illustrate the kind of results one can obtain from this general approach, we shall study one case explicitly.

4. The case of the sphere. On the unit sphere $S^{2}=\left\{(x, y, z) \in \mathbb{R}^{3}: x^{2}+y^{2}+z^{2}=\right.$ $1\}$, the generic point $P(x, y, z)$ has spherical coordinates $(\theta, \phi) \in[0, \pi] \times[0,2 \pi]$ with $x=\sin \theta \sin \phi, y=\sin \theta \cos \phi, z=\cos \theta$. The Riemannian measure is $d P=\sin \theta d \theta d \phi$. The Laplacian of $f: P \mapsto f(\theta, \phi)$ is given by $\Delta f=(\sin \theta)^{-1} \partial_{\theta}\left(\sin \theta \partial_{\theta} f\right)+(\sin \theta)^{-2} \partial_{\phi}^{2} f$. The distance from $P(\theta, \phi)$ to the north pole $N(0,0)$ is $r=d(P, N)=\theta$. The area and volume of a geodesic ball of radius $r$ are given by $A(r)=2 \pi \sin r$ and $V(r)=2 \pi(1-\cos r)$ respectively. Furthermore, $\delta=\pi$ and $V_{M}=V(\pi)=4 \pi$. The equality (5) leads to

$$
G_{2}(P, Q)=\frac{\log 2-1-\log (1-\cos [d(P, Q)])}{4 \pi}
$$

where $d(P, Q)$ is the distance between $P$ and $Q$ measured on the sphere. For each $\ell \in \mathbb{N}$, $-\ell(\ell+1)$ is an eigenvalue of the Laplacian with multiplicity $2 \ell+1$. The zonal and associated spherical functions are related to the Legendre polynomials and Legendre functions of the first kind, defined respectively by

$$
\begin{aligned}
p_{\ell}(x) & =\left(2^{\ell} \ell !\right)^{-1} \frac{d^{n}}{d x^{n}}\left(x^{2}-1\right)^{n}, \quad(\ell \in \mathbb{N}), \\
p_{\ell}^{m}(x) & =(-1)^{m}\left(1-x^{2}\right)^{m / 2} \frac{d^{m}}{d x^{m}} p_{\ell}(x), \quad(1 \leq m \leq \ell,-1 \leq x \leq 1) .
\end{aligned}
$$

An orthonormal basis of the eigenspace corresponding to the eigenvalue $-\ell(\ell+1)$ is given by the $2 \ell+1$ functions $\left\{f_{\ell}^{m}:-\ell \leq m \leq \ell\right\}$ defined, for $P=(\theta, \phi)$, by

$$
\begin{aligned}
f_{\ell}^{0}(P) & :=\left(\frac{2 \ell+1}{4 \pi}\right)^{1 / 2} p_{\ell}(\cos \theta), & (\ell \geq 0), \\
f_{\ell}^{m}(P) & :=\left\{\frac{(2 k+1)(\ell-m) !}{2 \pi(\ell+m) !}\right\}^{1 / 2} \cos (m \phi) p_{\ell}^{m}(\cos \theta), & (1 \leq m \leq \ell), \\
f_{\ell}^{m}(P) & :=\left\{\frac{(2 k+1)(\ell-|m|) !}{2 \pi(\ell+|m|) !}\right\}^{1 / 2} \sin (|m| \phi) p_{\ell}^{|m|}(\cos \theta), & (-\ell \leq m \leq-1) .
\end{aligned}
$$

$G_{2}$ has in $L^{2}$ the bilinear expansion

$$
G_{2}\left(P_{1}, P_{2}\right)=\sum_{\ell=1}^{\infty} \frac{f_{\ell}^{0}\left(\theta_{1}\right) f_{\ell}^{0}\left(\theta_{2}\right)}{\ell(\ell+1)}+\sum_{m \in \mathbb{Z}^{*}} \sum_{\ell=|m|}^{\infty} \frac{f_{\ell}^{m}\left(P_{1}\right) f_{\ell}^{m}\left(P_{2}\right)}{\ell(\ell+1)} .
$$

The average of $G_{2}\left(P_{1}, P_{2}\right)$ on the geographical parallel containing $P_{2}$ is, for $0<\theta_{1}, \theta_{2}<\pi$,

$$
\begin{aligned}
& \frac{2 \log 2-1-\log \left[1+\cos \min \left(\theta_{1}, \theta_{2}\right)\right]\left[1-\cos \max \left(\theta_{1}, \theta_{2}\right)\right]}{4 \pi} \\
& \quad=\sum_{\ell=1}^{\infty} \frac{f_{\ell}^{0}\left(\theta_{1}\right) f_{\ell}^{0}\left(\theta_{2}\right)}{\ell(\ell+1)}=\frac{1}{4 \pi} K_{A}\left(\frac{1-\cos \theta_{1}}{2}, \frac{1-\cos \theta_{2}}{2}\right)
\end{aligned}
$$

(see the last formula of $\S 5.4 .4$ in [MOS66] p. 239). Hence Anderson-Darling's kernel is the radial part of $G_{2}$, obtained by an averaging process. 
Note that $1-\cos [d(P, Q)]=\delta^{2}(P, Q) / 2$, where $\delta$ is the distance measured in $\mathbb{R}^{3}$. In view of (9) and [KB94], Theorem 4.3.1 p. 138, we obtain for the U-statistic with kernel $G_{2}$ the following result.

THEOREM 1. If $P_{1}, \ldots, P_{n}$ is a sample from the uniform distribution on $S^{2}$, one has the convergence in law

$$
-\frac{n}{4 \pi} \log \left(\frac{e g_{n}^{2}}{4}\right) \rightarrow \sum_{\ell=1}^{\infty} \frac{\chi^{2}(2 \ell+1)-(2 \ell+1)}{\ell(\ell+1)}
$$

where $g_{n}:=\prod_{1 \leq i<j \leq n} \delta\left(P_{i}, P_{j}\right)^{\frac{2}{n(n-1)}}$ is the geometrical mean of the distances between the points of the sample in $\mathbb{R}^{3}$, and $\left\{\chi^{2}(2 \ell+1)\right\}_{\ell \geq 1}$ denotes a sequence of independent $\chi^{2}$ random variables with $(2 \ell+1)$ degrees of freedom.

Extensions of this result to other 2-point homogeneous spaces will be discussed in a forthcoming paper.

\section{References}

[Ask75] R. Askey, Orthogonal Polynomials and Special Functions, Society for Industrial and Applied Mathematics, Philadelphia, Pa., 1975.

[Aub82] T. Aubin, Nonlinear Analysis on Manifolds. Monge-Ampère Equations, Grundlehren der Mathematischen Wissenschaften 252, Springer-Verlag, New York, 1982.

[Bes78] A. L. Besse, Manifolds All of Whose Geodesics are Closed, Ergebnisse der Mathematik und ihrer Grenzgebiete 93, Springer-Verlag, Berlin, 1978, With appendices by D. B. A. Epstein, J.-P. Bourguignon, L. Bérard-Bergery, M. Berger and J. L. Kazdan.

[DS86] R. B. D'Agostino and M. A. Stephens (eds.), Goodness-of-fit Techniques, Statistics: Textbooks and Monographs 68, Marcel Dekker Inc., New York, 1986.

[Dur73] J. Durbin, Distribution Theory for Tests Based on the Sample Distribution Function, Conference Board of the Mathematical Sciences Regional Conference Series in Applied Mathematics 9, Society for Industrial and Applied Mathematics, Philadelphia, Pa., 1973.

[Gra73] A. Gray, The volume of a small geodesic ball of a Riemannian manifold, Michigan Math. J. 20 (1973), 329-344.

[Hel00] S. Helgason, Groups and Geometric Analysis, Mathematical Surveys and Monographs 83, American Mathematical Society, Providence, RI, 2000, Integral geometry, invariant differential operators, and spherical functions, Corrected reprint of the 1984 original.

[KB94] V. S. Koroljuk and Yu. V. Borovskich, Theory of U-statistics, Mathematics and its Applications 273, Kluwer, Dordrecht, 1994.

[LS01] W. V. Li and Q.-M. Shao, Gaussian processes: inequalities, small ball probabilities and applications, in: Stochastic Processes: Theory and Methods, Handbook of Statist. 19, North-Holland, Amsterdam, 2001, 533-597.

[MOS66] W. Magnus, F. Oberhettinger, and R. P. Soni, Formulas and Theorems for the Special Functions of Mathematical Physics, Third enlarged edition, Grundlehren der mathematischen Wissenschaften 52, Springer-Verlag, New York, 1966. 
[SW86] G. R. Shorack and J. A. Wellner, Empirical Processes with Applications to Statistics, Wiley Series in Probability and Mathematical Statistics: Probability and Mathematical Statistics, John Wiley \& Sons, New York, 1986.

[Wan52] H. C. Wang, Two-point homogeneous spaces, Ann. of Math. (2), 55 (1952), 177-191. [Wat61] G. S. Watson, Goodness-of-fit tests on a circle, Biometrika 48 (1961), 109-114.

[Zol61] V. M. Zolotarev, Concerning a certain probability problem, Teor. Verojatnost. i Primenen. 6 (1961), 219-222. 